\title{
NECESIDAD DE FORMACIÓN DE PROFESIONALES EN EL ÁREA DE MANTENIMIENTO DE AERONAVES A NIVEL SUPERIOR EN HONDURAS
}

\author{
Iván-Vladimir Betancourt-Mendoza ${ }^{1, *}$, Ramón-Emilio Bueso-Carbajal ${ }^{1, \dagger}$ \\ ${ }^{1}$ Departamento de Ciencias Aeronáuticas, Facultad de Ciencias Espaciales, UNAH \\ Recibido: 04/diciembre/2019 \\ Aceptado: 21/febrero/2020 \\ DOI: https://doi.org/10.5377/ce.v12i2.10287
}

\begin{abstract}
RESUMEN
El presente artículo de investigación tuvo como objetivo analizar la necesidad de Formación de Profesionales en el área de Mantenimiento de Aeronaves a Nivel Superior en Honduras. Una propuesta académica en el tercer nivel educativo lograría un doble beneficio: el mejoramiento del estatus técnico y profesional de las personas que trabajan y trabajarán en el rubro del mantenimiento aeronáutico y el cumplimiento con estándares internacionales en la conformación de una propuesta formativa para este mismo rubro. La metodología aplicada incluyó, además de una revisión de las regulaciones aplicables al mantenimiento aeronáutico, una entrevista a especialistas con conocimiento de los ambientes académicos y prácticos de la ingeniería del mantenimiento aeronáutico y un grupo focal con técnicos activos. Entre los principales resultados se señalan de manera específica las limitaciones atribuibles al marco regulatorio nacional de Honduras y la forma en que estas limitaciones pueden ser complementadas con las orientaciones de la Organización de Aviación Civil Internacional - OACI a nivel mundial; también se recoge la anuencia por parte de técnicos y académicos para incorporar al rubro de mantenimiento, personal con una sólida formación físico matemática que debería ser impartida por la Universidad; un tercer resultado es una revisión de la oferta de carreras de grado para mantenimiento aeronáutico de un grupo selecto de universidades latinoamericanas y, un último resultado, una propuesta de malla curricular que toma en cuenta las orientaciones que se derivan de la información recolectada y cobra forma a la luz de la experiencia de los investigadores. La investigación concluye en la necesidad de contar en Honduras con una Carrera de Grado en Mantenimiento Aeronáutico para fortalecer en los profesionales de este campo y los potenciales estudiantes, las capacidades para aplicar en su trabajo criterios científicos que permitan una adecuada autonomía de decisión y la correcta aplicación de las directrices técnicas contenidas en los manuales técnicos del fabricante y las regulaciones de aeronáutica civil aplicables al campo del mantenimiento aeronáutico.
\end{abstract}

Palabras clave: Aeronáutica, técnico universitario, mantenimiento e ingeniería, malla curricular, capacitación

*ivan.betancourt@unah.edu.hn

†ramon.bueso@unah.edu.hn 


\begin{abstract}
This research article aimed to analyze the need for Professional Training in the area of Aircraft Maintenance at a Higher Level in Honduras. An academic proposal in the third educational level would achieve a double benefit: the improvement of the technical and professional status of the people working and they will work in the aeronautical maintenance sector and compliance with international standards in the conformation of a training proposal for this same item. The methodology applied included, in addition to a review of the regulations applicable to aeronautical maintenance, an interview with specialists with knowledge of the academic and practical environments of aeronautical maintenance engineering and a focus group with active technicians. Among the main results, the limitations attributable to the national regulatory framework of Honduras and the way in which these limitations can be complemented with the International Civil Aviation Organization - ICAO guidelines at international level are specifically noted; the consent of technicians and academics is also included to incorporate the personal maintenance field with a solid mathematical physical training that should be taught by the University; a third result is a review of the offer of degree careers for aeronautical maintenance of a select group of Latin American universities and, a final result is a proposal of curricular syllabus that takes into account the orientations that are derived from the information collected and takes shape in the light of the researchers' experience. The investigation concludes in the need to have in Honduras a Degree in aeronautical maintenance to strengthen in the professionals of this field and the potential new students, the abilities to apply scientific criteria in their work that allow an adequate autonomy of decision and the correct application of the technical guidelines contained in the technical manufacturer manuals and in the civil aeronautical regulations applicable to aeronautical maintenance.
\end{abstract}

Keywords: Aeronautics, maintenance, aeronautical management, specialize, supply and demand training.

\title{
1 Introducción
}

Actualmente, el enfoque moderno para el mantenimiento aeronáutico es sofisticado. Las aeronaves están diseñadas para cumplir con la seguridad operacional bajo la condición de aeronavegabilidad; para cumplir con lo anterior se desarrolla un programa de mantenimiento detallado junto con cada modelo de aeronave nueva o derivada de un modelo existente. Este programa de mantenimiento inicial puede ser adaptado por cada aerolínea para acomodarlo a la naturaleza de sus operaciones individuales. Esto asegura la operación continua de la aeronavegabilidad bajo cualquier circunstancia. Fabricantes, aerolíneas y agencias reguladoras trabajan para mejorar las técnicas de diseño y mantenimiento y con esto mantener a la industria de la aviación a la vanguardia en seguridad.

El cumplimiento de las tareas de mantenimiento en aviación, son ejecutadas por el Técnico encargado en la gestión del Mantenimiento Aeronáutico, por lo tanto, estos técnicos están directamente relacionados con el resguardo de la vida de todas las personas que viajan por vía aérea, incluyendo la tripulación, de ahí la importancia de su labor y la necesidad de contar con una formación profesional completa, para la optimización de su desempeño en el campo.

Se espera que este trabajo de investigación provea a la comunidad aeronáutica de información base para futuras investigaciones y se convierta en referente para la aplicabilidad de este proceso de formación profesional asegurando en alguna medida a los actuales y futuros técnicos en mantenimiento de aeronaves, una formación completa y en constante actualización. 
Como antecedentes podemos señalar: según datos de la OACI, para el 2030 existirá un déficit en la capacidad de entrenamiento de Técnicos en Mantenimiento de Aeronaves para formar una demanda de 360,000 técnicos alrededor del mundo hacia ese año; de hecho mientras ahora se cuenta con 52,260 centros de entrenamiento, para el 2030 serán necesarios 70,331, lo cual proyecta un déficit de 18,070 centros para dentro de tan solo 11 años (ICAO, 2011).

La des-categorización de Honduras en Seguridad Operacional de la Aviación por parte de evaluaciones del Programa de Evaluación de Seguridad de la Aviación Internacional (IASA por sus siglas en inglés) de la Administración Federal de la Aviación de los Estados Unidos de Norte América-FAA, tiene en cuenta que, la falta de formación de Técnicos en Mantenimiento Aeronáutico que cumplan con altos estándares de calidad en su desempeño que los posicionen a nivel internacional, sea una de las causas para que nuestro país no aparezca en el listado de países que cumplen con estándares internacionales en Seguridad Operacional Aeronáutica (FAA, 2019).

Por lo tanto, el cumplimiento con estos estándares es una de las principales orientaciones del trabajo del Departamento de Ciencias Aeronáuticas cuando desarrolla investigaciones para la conformación de la propia oferta académica y el desarrollo aeronáutico del País proponiendo este tipo de investigaciones.

Se pretende con el presente estudio, ofrecer una base informativa que llene el vacío de investigación percibido en el campo. Además, los análisis hechos aportaron elementos para la toma de decisiones en relación con el desarrollo de programas académicos en el campo del mantenimiento aeronáutico.

\section{Metodología}

Se hizo una revisión de distintos documentos regulatorios sobre la formación necesaria para un perfil de Profesionales en Mantenimiento Aeronáutico, incluyendo Regulaciones de Aeronáutica Civil aplicables y perfiles de puesto relacionados. Así mismo se revisaron planes de estudio de algunas de las universidades técnicas que cuentan con propuestas formativas relacionadas con el mantenimiento aeronáutico. De estas consultas se recogió información sobre número de espacios de aprendizaje propuesto, temas en mantenimiento aeronáutico, los créditos académicos de cada una de las propuestas y el tiempo de duración.

Durante la investigación se aplicó un grupo focal de profesionales y técnicos activos en funciones relacionados con el mantenimiento aeronáutico, hablando sobre las expectativas que tienen de un profesional aeronáutico con formación de grado o en alguna de las modalidades de educación no formal. Así mismo, se aplicó una encuesta en línea a profesionales relacionados con el mantenimiento de aeronaves.

Se realizaron dos entrevistas semiestructuradas mediante la aplicación de un cuestionario de 12 preguntas a dos expertos en temas de mantenimiento aeronáutico vinculado a procesos formativos en una universidad que cuenta con carreras aeronáuticas relacionadas.

Se aplicó un Benchmarking, a carreras de Mantenimiento Aeronáutico de las Universidades: Don Bosco (El Salvador), la Universidad Técnica Federico Santa María (Chile), la Universidad de Galileo (Guatemala), la Universidad Tecnológica de Panamá (Panamá) en donde se revisaron dos opciones de propuesta 
académica, así como la Universidad de Defensa de Honduras, UDH.

Como se aprecia en la figura 1, la comparación de las diferentes carreras generó un indicador del porcentaje de cada una de las clasificaciones que forman las diferentes asignaturas y que conlleva a apreciar que el $45 \%$ de la carrera deberá ser utilizada para las clases propias especificas del mantenimiento aeronáutico, un $18 \%$ para clases generales, $15 \%$ las clases de ingeniería, los demás se distribuyen casi equitativas en un $10 \%$ (diez por ciento) para las clases de idiomas, $8 \%$ (ocho por ciento) para las clases científicas y $4 \%$ (cuatro por ciento) para las clases de computación.

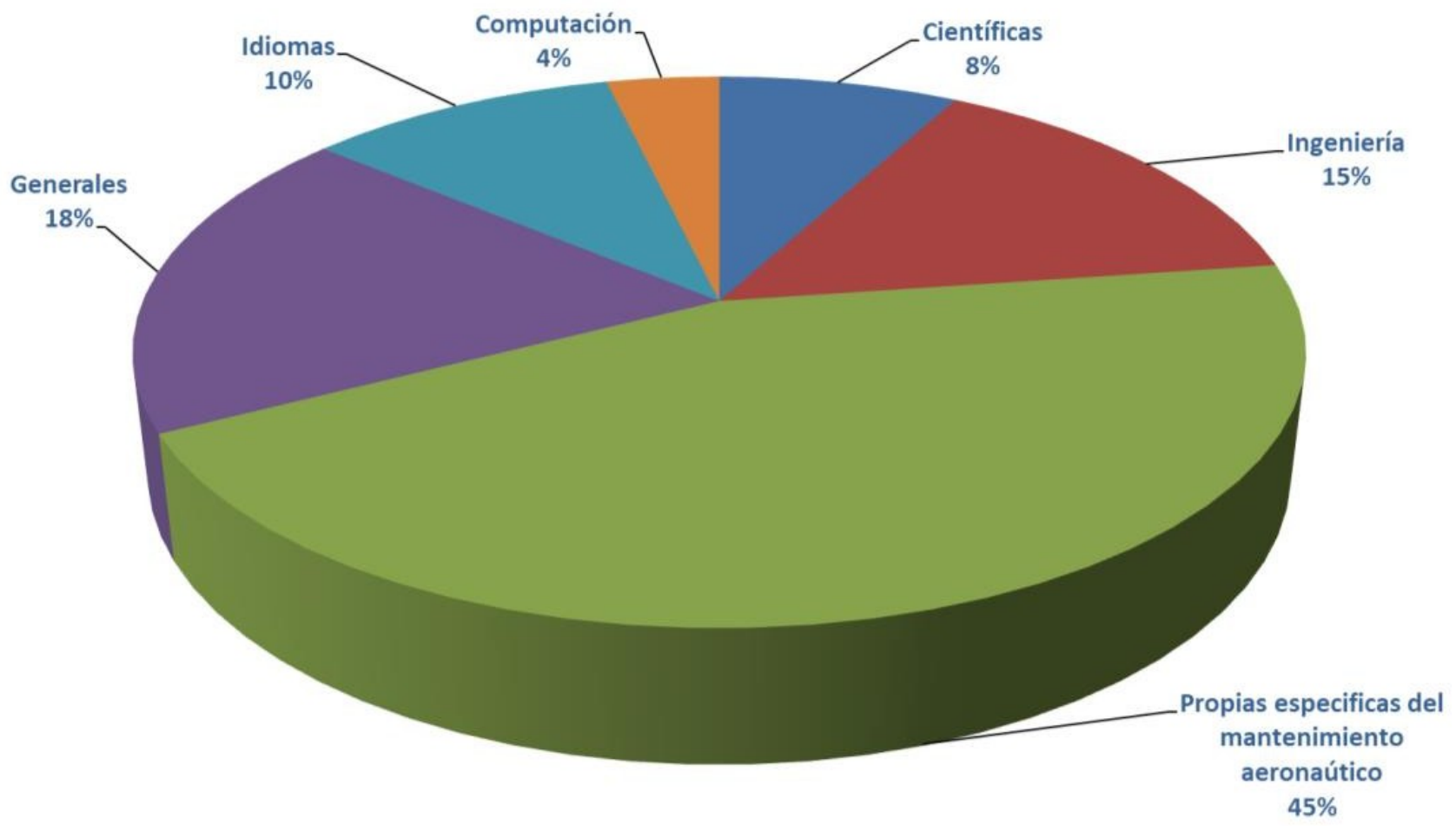

Figura 1: Ponderación de las tendencias educativas en las asignaturas de los técnicos en mantenimiento aeronáutico analizados.

En conclusión, se puede decir que, al hacer una revisión en los cinco Técnicos de las diferentes Universidades antes mencionadas a nivel del mantenimiento aeronáutico, se puede promediar en 35 clases aproximadamente de las que consta una carrera de técnico a nivel superior en mantenimiento de aeronaves.

\section{Resultados}

\section{Marco Regulatorio}

1.1 Existe en nuestro País la Ley de Aeronáutica Civil la cual se ejecuta en su parte operativa a través de las Regulaciones de Aeronáutica Civil-RACs (AHAC, 2019). En este marco encontramos las Regulaciones: Licencias al Personal Técnico Aeronáutico-LPTA y, la RAC 147-Organización de Formación de Mantenimiento Aprobada, las cuales se refieren a los temas en los cuales debe estar capacitado un técnico en mantenimiento de aeronaves TMA. Sin embargo, la información que se brinda es general y no especifica aspectos como, los campos del conocimiento 
físico-matemático, el dibujo técnico aeronáutico, el inglés técnico o computación; conocimientos básicos que el personal antes mencionado debe adquirir y poseer al egresar de un proceso formativo acorde con el campo a desempeñarse. ${ }^{1}$

1.2 Complementando las regulaciones hondureñas el Documento 7192-Manual de Instrucción del Técnico en Mantenimiento de Aeronaves de la Organización de Aviación Civil Internacional (ICAO, 2003), hace una propuesta formal en cuanto a la fundamentación físico-matemática especifica requerida, así como las materias de la parte propiamente técnica del mantenimiento de aeronaves, encontrándose o siendo notorio que la OACI en su documento refleja un horizonte de pensamiento universitario por el alcance de las materias ahí enunciadas para conformar un pensum académico de un TMA. Esto hace que este documento pueda ser tomado como base para la construcción de una malla curricular propuesta para un Técnico Universitario en Mantenimiento Aeronáutico-TUMA.

2. Aportes de los gerentes y jefes de área, así como especialistas académicos.

2.1 Los gerentes de área, específicamente de mantenimiento y calidad, expresan que sería ideal contratar personal ya formado con las bases del mantenimiento aeronáutico que podría brindar la UNAH en una carrera de Técnico Universitario, ven la necesidad de formación general en mantenimiento de aviación antes de contratarlos, para después la empresa darles formación específica en el equipo (modelo de aeronave) a trabajar. Actualmente ellos contratan técnicos con muy poca o ninguna formación académica en mantenimiento de aviación y esto redunda en que la empresa debe capacitarlos primero en conocimientos generales, en procedimientos y prácticas estándar del mantenimiento de aviación, en como leer y entender manuales técnicos de fabricantes de productos aeronáuticos, así como en Regulaciones de Aeronáutica Civil; conocimientos que pueden ser adquiridos antes en un centro de formación académica universitaria, para que después, la empresa los forme en campos técnicos específicos de acuerdo con las aeronaves que operan. Esto le daría una mejor producción en el campo del mantenimiento aeronáutico a la empresa, optimizando el presupuesto asignado a este rubro, ya que los técnicos entrarían directamente a recibir entrenamiento en el modelo de aeronave específico en el que trabajarían, ya con la sólida formación universitaria en mantenimiento aeronáutico.

2.2 Con respecto a los conocimientos básicos, expresan que los técnicos adolecen, además de la capacitación académica base para trabajar en aviación, de los conocimientos en las áreas de matemáticas, física, inglés y computación para ser aplicados en el mantenimiento aeronáutico, así como de la capacidad de leer planos estructurales y eléctricos en manuales técnicos emitidos por el fabricante de los productos aeronáuticos (aeronave, motor, hélice). Esta opinión es generalizada y se ve expresada a lo largo del conjunto de entrevistas realizadas. Lo anterior coincide con las falencias encontradas en las regulaciones relacionadas y que se mencionan en el párrafo 1.1 del presente artículo.

2.3 Una de las observaciones importantes hechas por los entrevistados se refiere al tema de acordar con la AHAC el hecho de que al egresar del proceso formativo del TUMA, el alumno pueda optar a tramitar su licencia de Técnico en Mantenimiento de Aeronaves T-II de acuerdo con lo requerido en el RAC LPTA 240(a)(3)(ii) (AHAC, 2019) en donde se estable que "Cuando el solicitante se halla graduado de una escuela aprobada por la AHAC, el solicitante debe poseer por lo menos 12

\footnotetext{
${ }^{1}$ Para revisar información específica sobre un perfil técnico, Véase el RAC 147.120 Material de formación de mantenimiento y RAC 147.200 Curso de formación básica aprobado; así como RAC LPTA 220 Licencia de Mecánico de a Bordo, RAC LPTA 240 Licencia Técnico de Mantenimiento de aeronaves tipo II. (TMA II) y RAC LPTA 245 Técnico de mantenimiento de aeronaves (TMA) tipo I.
} 
meses de experiencia trabajando para un organismo de mantenimiento aprobado bajo supervisión". En este caso el tiempo que se propone dure la carrera en la UNAH (2 años y medio), podría llenar el requisito anterior en cuanto a la experiencia exigida, ya que los alumnos harán las prácticas correspondientes en organizaciones de mantenimiento aprobado durante los periodos académicos de la carrera.

3. Oferta Académica Regional de Técnicos en Mantenimiento Aeronáutico

En nuestra región centroamericana existen universidades que brindan carreras en mantenimiento aeronáutico, las cuales podemos citar:

- La Universidad Don Bosco en El Salvador que oferta una carrera con Título de Técnico/a en Mantenimiento Aeronáutico con duración de 2 años (4 ciclos), 23 asignaturas y 113 unidades valorativas;

- La Universidad Galileo en Guatemala que ofrece la carrera Técnico Universitario en Mecánica de Aviación con una duración de 12 ciclos (4×año), 60 asignaturas y 180 créditos académicos;

- La Universidad Tecnológica de Panamá que oferta la carrera de Técnico en Ingeniería de Mantenimiento de Aeronaves con Especialidad en Motores y Fuselaje con una duración de 3 años, 43 asignaturas y 120 créditos académicos.

Al comparar los pensum académicos de estas universidades, se pudo notar que los componentes profesionalizantes del campo del mantenimiento aeronáutico, así como la base físico-matemática, el idioma inglés y computación son los campos del conocimiento que conforman la base de la malla curricular de un Técnico Universitario en Mantenimiento Aeronáutico.

4. Propuesta de una malla curricular en nuestro País.

\subsection{Los técnicos en la UNAH}

Antes de la conformación de un Técnico Universitario o Tecnólogo en Mantenimiento Aeronáutico en la UNAH, se decidió investigar a lo interno como están conformadas las carreras técnicas actuales en cuanto a su número de espacios de aprendizaje, sus créditos académicos totales, así como los créditos académicos profesionalizantes o del campo del conocimiento específico (UNAH, 2019), lo anterior para poder determinar qué tanto es el peso o ponderación de estos últimos en la malla curricular con respecto al total de los créditos académicos. A continuación, se muestra la gráfica del análisis efectuado: 


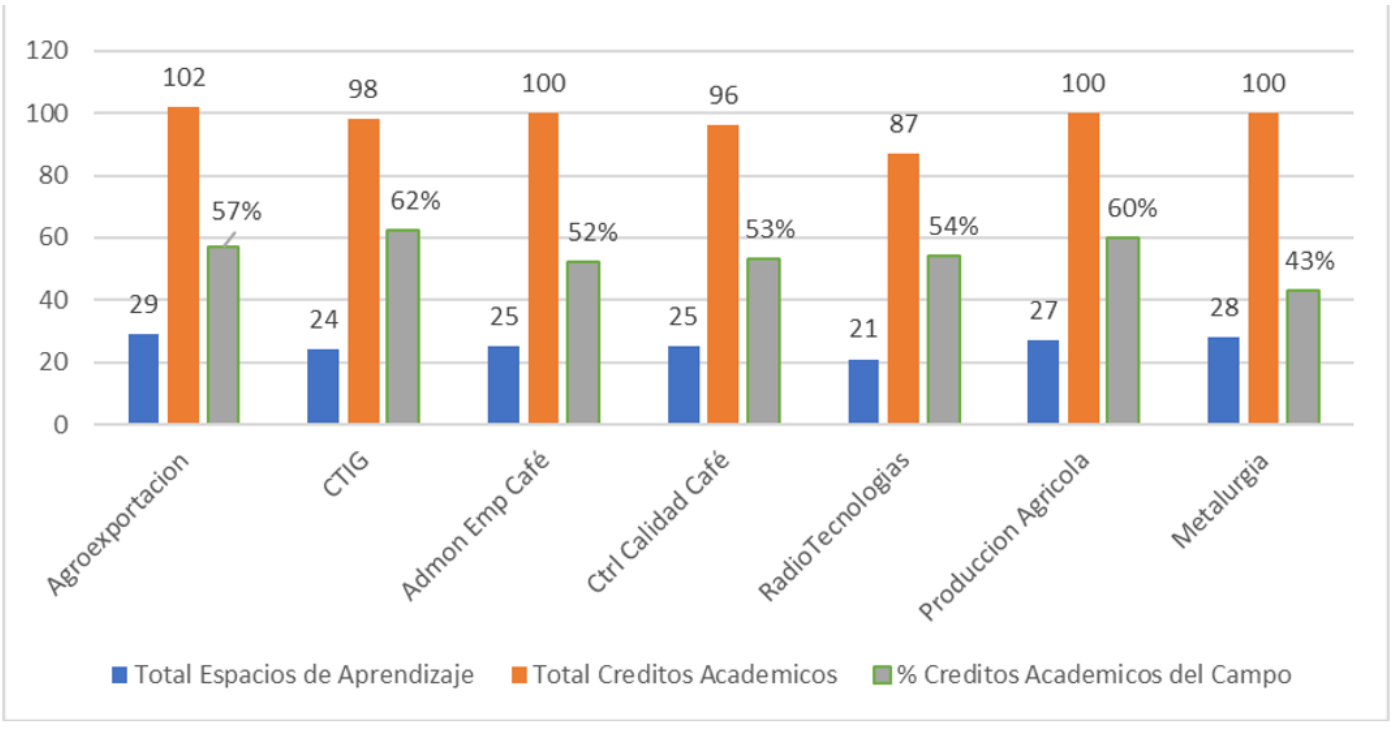

Figura 2: Datos curriculares de carreras técnicas en la UNAH.

Se puede observar que, para un técnico universitario de la UNAH, los créditos académicos (C.A.) andan en un promedio aproximado de 98 C.A. También se observa que los espacios de aprendizaje tienen un promedio aproximado de 26 Espacios de Aprendizaje (E.A.). Con respecto a la relación de los créditos profesionalizantes a los créditos totales de la carrera, tenemos un porcentaje promedio aproximado de $54 \%$. Este porcentaje es el peso académico del campo del conocimiento específico que se brinda a un Técnico Universitario en la UNAH según esta muestra de siete carreras técnicas.

Se debe hacer notar que existen otras carreras técnicas que brinda la UNAH, pero estas se salen del comportamiento normal de la mayoría en cuanto a las estadísticas antes mencionadas y por eso no se tomaron en cuenta en el estudio.

Otra anotación relevante revisando los planes de estudio, es el caso de carreras técnicas que, aunque pueden tener un componente aparentemente no tan alto en C.A. profesionalizantes, los espacios de aprendizaje relacionados ocupan como requisito una base físico-matemática significativa para poder desarrollarse. Ejemplo de esto es el Técnico en Metalurgia que tiene $43 \%$ de C.A. profesionalizantes, pero tiene $23 \%$ C.A. en el componente físico-matemático.

\subsection{Malla curricular propuesta}

\subsubsection{Malla Propuesta}

La OACI en su Doc. 7192 recomienda se impartan pericias manuales e intelectuales, así como conocimientos sólidos de la teoría físico-matemática y un conocimiento completo de la aeronave o del sistema específico en el que tendrán que trabajar (ICAO, 2003).

En el caso de la Matemática de acuerdo con lo recomendado, los escenarios podrían variar debido a que los contenidos son amplios, panorama que va desde aritmética hasta ecuaciones diferenciales pasando por álgebra, geometría y trigonometría, así como cálculo. 
Como un aporte de la Academia a la creación del TUMA, debemos señalar que, según la experiencia en el campo del mantenimiento aeronáutico en el país, se considera que el TUMA debe conocer como calcular e inferir en base a la Estadística, las proyecciones del control y la planificación del mantenimiento aeronáutico para la óptima gestión de la flota y, por lo tanto, las operaciones de la aerolínea.

Por otro lado, el tema de Programación también es considerado un aporte de la academia en base a la experiencia del investigador en el campo, ya que el TUMA debe conocer como interactuar con las computadoras de abordo de la aeronave, especialmente con las que graban los datos del desempeño y operación de los sistemas de propulsión, así como con las que muestran las fallas de los sistemas electromecánicos de la aeronave y conocer como leer e interpretarlas para su evaluación en tierra. Esta información es muy útil para el TUMA en el diagnóstico de hallazgos y corrección de fallas, así como para la confirmación del correcto funcionamiento de los sistemas en general. También es necesario con alguna frecuencia, actualizar con nuevo software las computadoras de abordo y, en algunos casos, reprogramar dichas computadoras, para lo cual el TUMA debe estar capacitado.

Por estas razones se decide incluir los espacios de aprendizaje, MM-100 Introducción a la Estadística y MM-314 Programación en la base matemática de la malla curricular propuesta del TUMA.

Por último, debemos anotar que se ofrecen tres (3) campos del conocimiento optativos para el TUMA a cursarse en los dos últimos periodos: Motores Aeronáuticos, Electricidad e Instrumentos Aeronáuticos, y Aviónica. Las opciones tomadas por el estudiante marcaran una orientación de la carrera de entre las tres (3) posibilidades de elección antes mencionadas.

A continuación, y tomando en cuenta las necesidades nacionales según las personas entrevistadas los pensum académicos consultados, las carreras técnicas de la UNAH y las orientaciones OACI del Doc. 7192 así como el aporte de la academia, se propone a continuación una malla curricular base: 


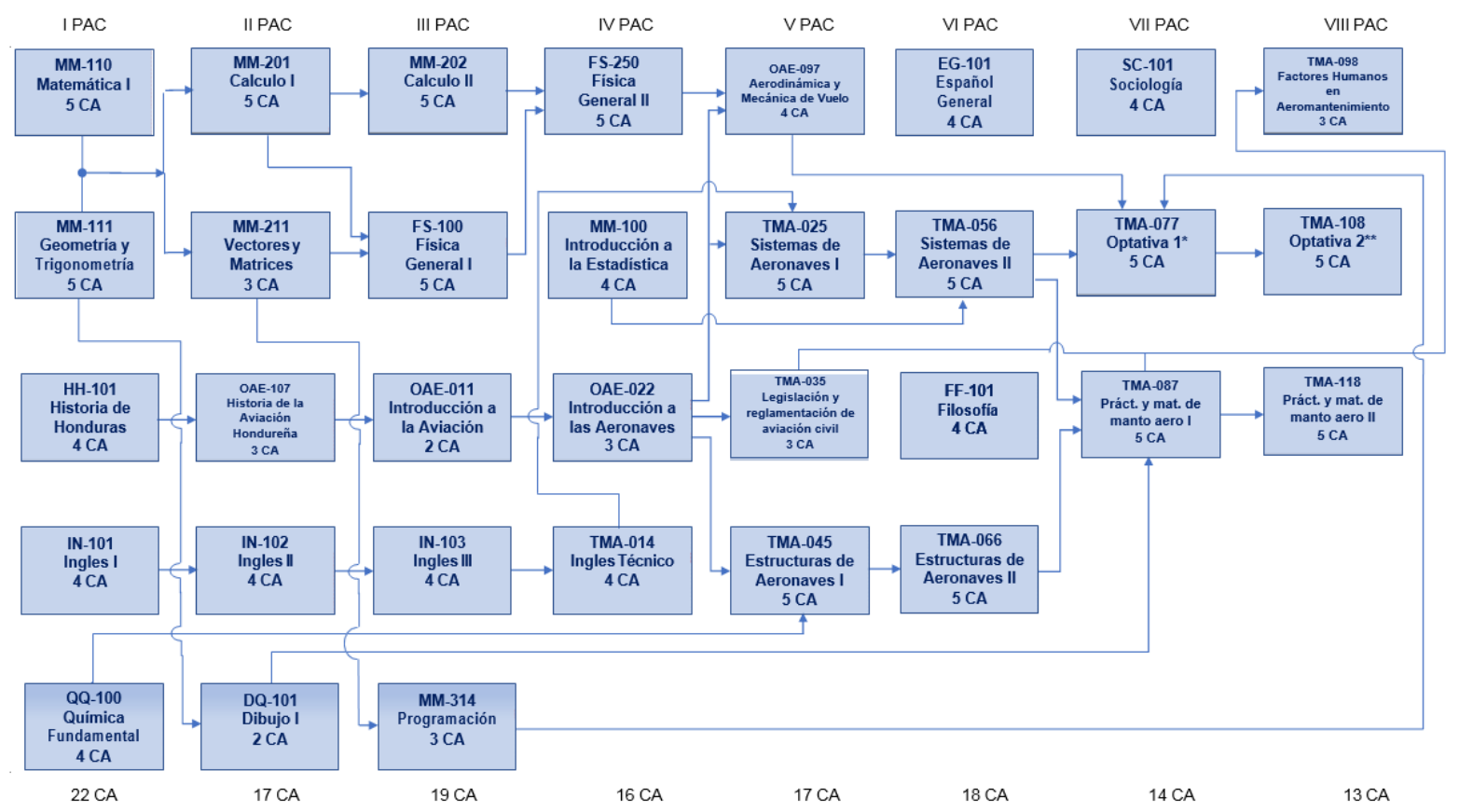

Figura 3: Flujograma de la primera aproximación de la carrera Técnico Universitario Mantenimiento Aeronáutico.

El estudiante seleccionará dos asignaturas optativas de acuerdo con una rama diversificada, según los siguientes campos:

Tabla 1: Ramas diversificadas del TUMA

\begin{tabular}{|c|c|c|c|c|c|}
\hline $\mathbf{N}^{\circ}$. & Código & Espacio de aprendizaje & C.A. & Requisitos & Campos \\
\hline 1 & *TMA-077 & Sistemas motopropulsores I & 5 & $\begin{array}{l}\text { TMA-056, } \\
\text { OAE-097, } \\
\text { MM-314 }\end{array}$ & Motores aeronáuticos \\
\hline 2 & **TMA-098 & Sistemas motopropulsores II & 5 & TMA-077 & Motores aeronáuticos \\
\hline \multirow[t]{2}{*}{1} & *TMA-077 & $\begin{array}{l}\text { Sistemas eléctricos e instru- } \\
\text { mentos I }\end{array}$ & 5 & TMA-056, & Electricidad e Instrumentos Aeronáuticos \\
\hline & & & & $\begin{array}{l}\text { OAE-097, } \\
\text { MM-314 }\end{array}$ & \\
\hline 2 & **TMA-098 & $\begin{array}{l}\text { Sistemas eléctricos e instru- } \\
\text { mentos II }\end{array}$ & 5 & TMA-077 & Electricidad e instrumentos aeronáuticos \\
\hline 1 & *TMA-077 & SCAV-navegación/radio I & 5 & $\begin{array}{l}\text { TMA-056, } \\
\text { OAE-097, } \\
\text { MM-314 }\end{array}$ & Aviónica \\
\hline 2 & **TMA-098 & SCAV-navegación/radio II & 5 & TMA-077 & Aviónica \\
\hline
\end{tabular}


La distribución porcentual de los campos del conocimiento y créditos académicos que constituyen el TUMA se ve reflejada en la siguiente gráfica:

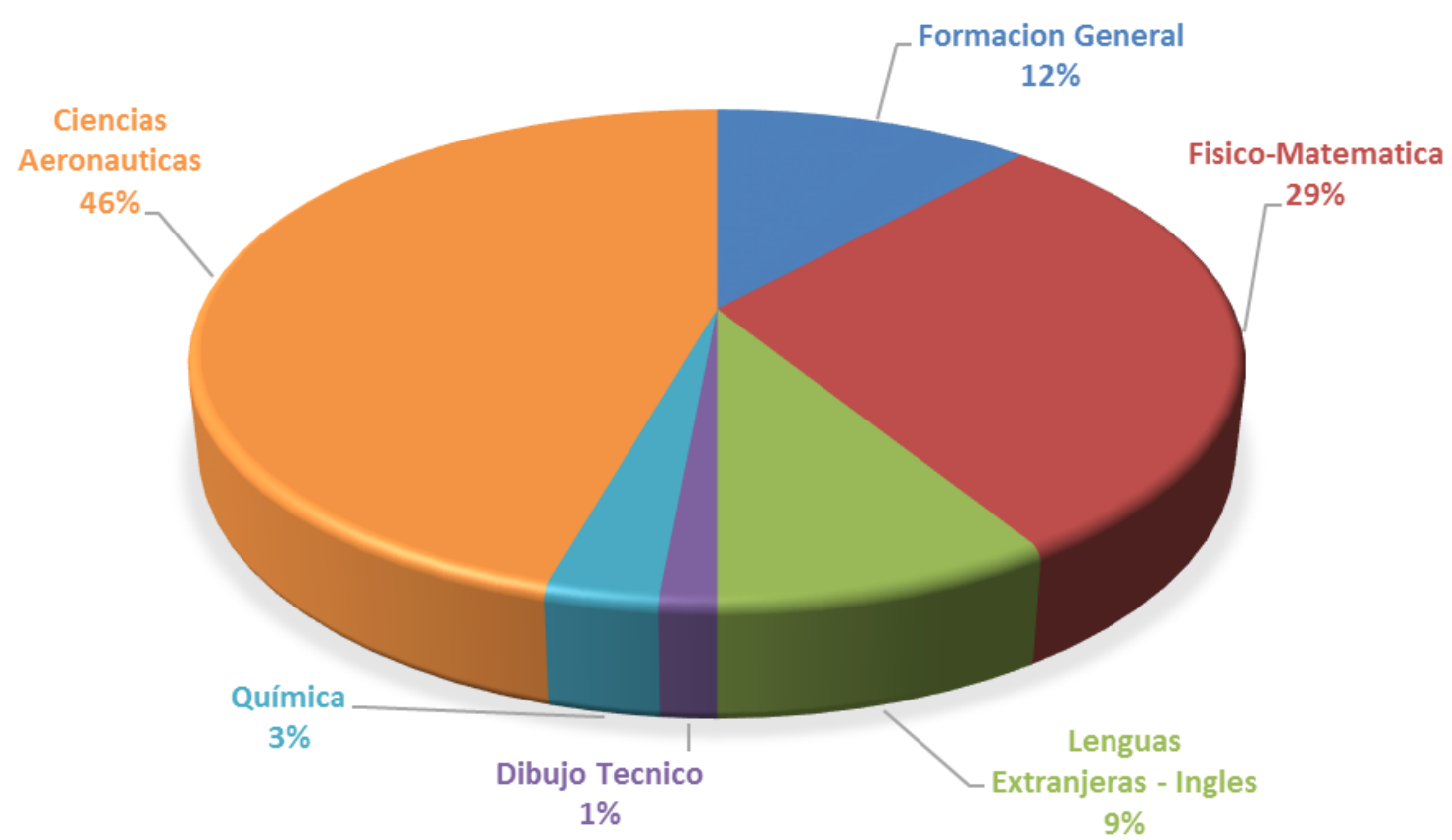

Figura 4: Distribución de C.A. por campo del conocimiento del TUMA.

En la gráfica podemos observar que se cuenta con $46 \%$ de créditos académicos profesionalizantes o específicos del campo, pero a la vez se cuenta con $29 \%$ del componente físico matemático; parámetros que se manejan en la UNAH en cuanto a datos curriculares de carreras técnicas.

Hay que hacer notar que el componente de lenguas extranjeras en la figura 4 se observa de un $9 \%$ el cual corresponde a los espacios de aprendizaje de Ingles I, II y III, sin embargo, si se revisa la malla curricular, observamos que existe un cuarto inglés, el Inglés Técnico (nivel IV OACI), el cual forma parte del $46 \%$ del componente de ciencias aeronáuticas.

4.2.2 Variables de la malla curricular según el componente matemático.

Esta propuesta variable de la malla curricular se expone para tratar de abarcar en su totalidad la recomendación de la OACI en cuanto a que un técnico en mantenimiento aeronáutico debe conocer las aplicaciones básicas de las ecuaciones diferenciales en el campo físico práctico, pero debe valorarse el hecho también de que el componente matemático seria aumentado con respecto a un Técnico Universitario.

En este caso el componente de Ecuaciones Diferenciales aplicado a las Ciencias Aeronáuticas es lo que haría variar la malla curricular en el bloque físico-matemático. Aunque existe ya en la UNAH una clase con este tema, MM-411 Ecuaciones Diferenciales, su temática es muy amplia e ingenieril y no es aplicable tal cual se propone para el TUMA. 
Para cubrir esta recomendación de capacitación, se propone crear en el DCA un nuevo espacio de aprendizaje con cuatro áreas temáticas o unidades de aprendizaje. Dicho E.A. tendría en sus dos primeras unidades las bases de las ecuaciones diferenciales en lo que respecta a ecuaciones diferenciales ordinarias y en derivadas parciales, y en las dos unidades restantes las aplicaciones en el campo aeronáutico. Este E.A. se agregaría a la malla curricular existente, con 3 créditos académicos y como requisito o pre-requisito tendría el E.A. MM-202 Cálculo II.

\section{Discusión}

La comparación de las diferentes carreras generó un indicador del porcentaje de cada una de las clasificaciones que forman las diferentes asignaturas y que conlleva a apreciar que el $45 \%$ de la carrera deberá ser utilizada para las clases propias especificas del mantenimiento aeronáutico, un $18 \%$ para clases Generales, $15 \%$ las Clases de Ingeniería, el resto de contenidos se distribuyen casi equitativas en un $10 \%$ (diez por ciento) para las clases de Idiomas, $8 \%$ (ocho por ciento) para las clases Científicas y $4 \%$ (cuatro por ciento) para las clases de Computación.

Los encuestados fueron representantes de empresas que desarrollan o han desarrollado actividades directas con la Aeronáutica Civil, tienen presencia en el mercado aeronáutico por más de 5 años, han tenido a su cargo personal laboral y cuentan con algún tipo de capacitación de mantenimiento de aeronaves y que pudieran ser considerados posibles contratantes de egresados como profesionales en el área de mantenimiento de aeronaves a nivel superior en Honduras.

El $89 \%$ de los encuestados opinan que es "Muy importante" y un 11 \% restante opinan que es "importante" que haya Formación Profesional a nivel Superior en el área de Mantenimiento de Aeronaves. Un 89 \% de los encuestados consideran que existe una demanda real de contratación de profesionales con formación en el área de mantenimiento de aeronaves a nivel superior. El 85\% de los entrevistados considera que la institución en la que labora estaría dispuesta a contratar profesionales con formación en el área de mantenimiento de aeronaves a nivel superior.

En base a lo anterior se puede inferir que efectivamente existe demanda, descrita por parte de los entrevistados que laboran en el ámbito aeronáutico señalando que hay una necesidad de formación profesional a nivel Superior en el área de mantenimiento de aeronaves en Honduras.

\section{Conclusiones}

1. Considerando las consultas hechas y el conocimiento acumulado del investigador se constata la necesidad de contar en Honduras con Técnicos en Mantenimiento Aeronáutico con conocimientos que les permitan desempeñarse con autonomía y competencias en el cumplimiento de directrices y procedimientos técnicos, que requieren el entendimiento y el dominio de terminología, conocimiento profundo del campo y el manejo de destrezas y pericias propias de la ingeniería del mantenimiento aeronáutico. 
2. Las universidades consultadas de la región presentan Carreras de Grado con similitudes suficiente como para proponerse como un referente en la conformación de un técnico universitario concebido para el ámbito hondureño; además, el repaso de las carreras revisadas permitió visualizar los extremos de una malla curricular, que elimina elementos esenciales de la carrera o incorpora un número excesivo de espacios de aprendizaje para un técnico universitario.

3. Para una primera aproximación a la malla curricular de un Técnico en Mantenimiento Aeronáutico, se deben incorporar las orientaciones derivadas de al menos tres fuentes:

a. El Documento 7192 de OACI (ICAO, 2003) sobre formación de técnicos en mantenimiento aeronáutico;

b. Consulta a directores de mantenimiento, así como a técnicos activos en el mantenimiento aeronáutico $\mathrm{y}$,

c. La experiencia de campo del investigador en términos de aplicación de criterios en la selección de espacios de aprendizaje y contenidos necesarios para una combinación pedagógica adecuada y pertinente.

4. Se pudo detectar que existen tres ramas o campos importantes en los cuales el técnico puede decidir diversificar su especialidad técnica estas son:

a. Sistema eléctrico e instrumento

b. Propulsión (motores)

c. Aviónica (AP, navegación, radio)

5. Se constató en toda la investigación la importancia del área curricular físico matemática para la formación de un técnico en mantenimiento; los espacios de aprendizaje propios de esta área ocupan una porción importante en todas las mallas curriculares revisadas. Por su parte el Documento 7192 de OACI recomienda explícitamente los contenidos de la Matemática y de la Física, así como el Dibujo Técnico y la Química en los que el proceso formativo debe hacer énfasis (ICAO, 2003).

\section{Referencias}

AHAC (2019). Agencia Hondureña de Aeronautica Civil-AHAC. Obtenido de Regulaciones Aeronáuticas: http://ahac.gob.hn/regulaciones-aeronauticas/.

FAA (2019). Federal Aviation Administration-FAA. Obtenido de International Aviation Safety Assessment (IASA) Program: https://www.faa.gov/about/initiatives/iasa/.

ICAO (2003). Manual de instrucción del técnico en mantenimiento de aeronaves-doc 7192. Montreal, Canada.

ICAO (2011). Global and regional 20-year forecasts. Montreal: ICAO.

UNAH (2019). Direccion del Sistema de Admision. Obtenido de Oferta Académica-UNAH: https: / / www. unah. edu.hn/oferta-academica. 